\title{
ESTUDIO FARMACOCINÉTICO COMPARATIVO ENTRE DIAZEPAM Y MIDAZOLAM, POST ADMINISTRACIÓN INTRAVENOSA E INTRAMUSCULAR EN AVESTRUZ DOMÉSTICO(Struthio camelus)
}

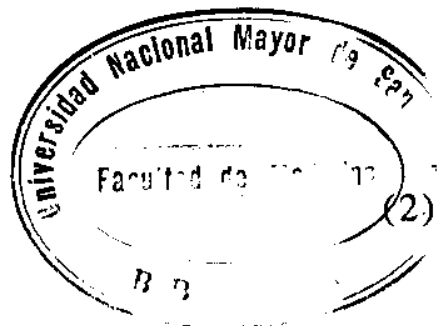

\author{
Luis Olivos $\mathbf{O}^{1}$, José Juárez $\mathbf{E}^{1}$, Julio De Lucas $B^{2}$ \\ (1) Facultad de Farmacia y Bioquímica UNMSM
}

2) Facultad de Veterinaria.Universidad Complutense de Madrid

\section{RESUMEN}

El interés en el manejo de especies animales con fines experimentales y de estudios farmacocinéticos, por la necesidad de pautas terapéuticas más racionales, nos llevó a desarrollar el presente estudio. Se utilizaron seis avestruces juveniles sanos, a los que se administró diazepam (DZP) y midazolam (MDZ) vía IV e IM, a dosis única de $0,5 \mathrm{mg} / \mathrm{kpv}$. En cuanto a DZP, la cuantificación por HPLC/UV(220nm) de los parámetros farmacocinéticos post administración IV, nos dieron los siguientes valores: MRTi=71,17 $\pm 29,97 \mathrm{~min}$, $\mathrm{t}_{1 / 2} \beta=49,30 \pm 21,18 \mathrm{~min}, \quad \mathrm{Cli}=87 \pm 50 \mathrm{~mL} / \mathrm{kg} / \mathrm{min}$ y $\mathrm{Vc}=1,82 \pm 0,35 \mathrm{~L} / \mathrm{kpv} ;$ mientras que las concentraciones plasmáticas post administración IM, no permitieron el tratamiento cinético. Para MDZ los parámetros farmacocinéticos post administración IV e IM corresponden respectivamente a $M R T i=59,70 \pm 37,14$ y $50,09 \pm 12,71 \mathrm{~min}, \quad t_{1 / 2} \beta=39,64 \pm 25,30$ y $35,32 \pm 10,93 \mathrm{~min}, \mathrm{Cli}=37 \pm 20 \mathrm{~mL} / \mathrm{kg} / \mathrm{min}$ y $\mathrm{Vc}=0,72 \pm 0,24 \mathrm{~L} / \mathrm{kpv}$. La biodisponibilidad post administración IM fue $99,97 \pm 30,43 \%$ con $C \max =0,36 \pm 0,09 \mu \mathrm{g} / \mathrm{mL}$ y $\operatorname{tmax}=11 \pm 2,24 \mathrm{~min}$. No se detectaron concentraciones plasmáticas de 1-hidroxi-midazolam (metabolito activo). Existe una correlación entre los datos de laboratorio obtenidos y las observaciones clínicas in situ. Se concluye que DZP no fue una opción válida para tranquilización o inducción anestésica en avestruces juveniles. MDZ sí demostró una depresión del SNC significativa por ambas vías.

La técnica analítica utilizada es confiable de acuerdo a los parámetros de validación empleados.

palabras claves: Avestruz. farmacocinética. hplc. tranquilización. anestesia. midazolam. diazepam. 


\section{ABSTRACT}

Our interest in handling animal species with the purpose of experimentation and pharmacokinetic study, due to the necessity of more rational guidelines, have motivated us to develop the present study in six healthy young ostriches to whom we administered diazepam (DZP) and midazolam (MDZ) via IV and IM in a simple dose of $0,5 \mathrm{mg} / \mathrm{kg}$. By HPLC/u.v quantification $(220 \mathrm{~nm})$ we observed that pharmacokinetics parameters of DZP after IV administration, corresponded to $\mathrm{MRTt}=71,17 \pm 29,97 \mathrm{~min}, \mathrm{t}_{1 / 2} \beta=49,30 \pm 21,18 \mathrm{~min}$, $\mathrm{Cl}=87 \pm 50 \mathrm{~mL} / \mathrm{kg} / \mathrm{min}$ and $\mathrm{Vcc}=1,82 \pm 0,35 \mathrm{~L} / \mathrm{kg}$. The plasma DZP concentrations post IM administration and the two main active metabolites concentrations after IV/IM administration did not allow a kinetic treatment of them. The pharmacokinetics parameters of MDZ after IV and $\mathrm{IM}$ administration respectively corresponded to $\mathrm{MRTi}=59,70 \pm 37,14$ and $50,09 \pm 12,71 \mathrm{~min}$, $\mathrm{t}_{1 / 2} \beta=39,64 \pm 25,30$ and $35,32 \pm 10,93 \mathrm{~min}, \mathrm{Cli}=37 \pm 20 \mathrm{~mL} / \mathrm{kg} / \mathrm{min}$ and $\mathrm{Vc}=0,72 \pm 0,24 \mathrm{~L} / \mathrm{kg}$. The bioavailability after IM administration was $99,97 \pm 30,43 \%$ with $\mathrm{Cmax}=0,36 \pm 0,09 \mu \mathrm{g} / \mathrm{mL}$ and $\operatorname{tmax}=11 \pm 2,24 \mathrm{~min}$. The plasma concentrations of 1-hidroxi-midazolam (active metabolite) were not detected. A correlation between the laboratory data and the clinical observations in situ have been stablished. We conclude that DZP was not a valid option for treatment of anxiety or anesthetic induction in young ostriches. On the other hand, MDZ demonstrated a significant depression of the CNS, by both ways. The technique used was reliable according to the validation parameters employed.

key words: ostrich. pharmacokinetic. hplc. tranquilization. anaesthesia. midazolam. diazepam.

\section{INTRODUCCIÓN}

Los avestruces domésticos en su edad adulta alcanzan gran fuerza y tamaño, muestran un carácter asustadizo y en general actúan de forma poco predecible. Esto hace que bajo situaciones de estrés reaccionen de forma incontrolada, pudiendo causar lesiones a sí mismos, a otros avestruces o a los criadores. Su cría en régimen semiextensivo, dificulta considerablemente el manejo cuando es necesario realizar una contención del ave con finalidad diagnóstica o terapéutica ${ }^{2}$.
Actualmente no existe ningún sistema físico o farmacológico que permita una contención eficaz del avestruz, con total garantía de seguridad para el ave y para el veterinario. Se han ensayado diferentes fármacos que incluyen agonistas $\alpha_{2}$ (xilacina, medetomidina, romifidina), benzodiacepinas (diazepam, midazolam), barbitúricos (pentobarbital), agentes esteroidales (alfadolona-alfaxolona) y opiáceos (carfentanilo), con objeto de sedar, inmovilizar o anestesiar avestruces, emúes y ñandúes, sin que ninguno de éstos, empleados de forma aislada, hayan 
producido los efectos deseados ${ }^{3,7,8,9}$. Las benzodiacepinas presentan efecto ansiolítico, hipnótico, anticonvulsivante, relajante muscular y amnésico anterógrado. Facilitan el manejo, inducen efecto de doma, reducen el miedo, la ansiedad y en cierta medida el estrés de origen central. Administrados por diferentes vías (IV, IM, PO.) son ampliamente empleadas para inducción, sedación y mejorar la anestesia en especies exóticas y domésticas, en combinación con otros agentes depresores del SNC. Su dosis efectiva depende, no sólo de la vía de administración elegida, las características individuales del paciente (requerimientos metabólicos, influencia del sistema porta renal, porcentaje de nefronas reptilianas, etc), ${ }^{5}$ sino de la aplicación única o en combinación con otros depresores del SNC. Los estudios farmacocinéticos son esenciales para practicar una correcta dosificación de los fármacos, porque la extrapolación de datos de otras especies pueden determinar que se alcancen niveles subterapéuticos o tóxicos.

El objetivo del presente estudio ha sido poner a disposición de la comunidad científica información sobre la evolución de los niveles plasmáticos en avestruces domésticos, respecto al tiempo, observados post-administración de diazepam y midazolam, de su biodisponibilidad post administración IM, así como algunas observaciones clínico-veterinarias de los efectos. De este modo el clínico puede seleccionar el fármaco y la vía más adecuada, estableciendo pautas de dosificación e intervalos acordes con el objetivo de su actuación clínica, con garantía de seguridad y eficacia, basándose en criterios científicos y contrastándolos con la experiencia de otros países con igual o diferentes especies animales y regímenes de crianza.

\section{MATERIAL Y MÉTODOS}

El trabajo fué realizado en la Cátedra de Farmacología Terapéutica y Farmacia de la Facultad de Veterinaria de la Universidad Complutense de Madrid (España), utilizando 6 avestruces domésticos de producción cárnica, de 7 meses de edad, criados en el criadero experimental de la misma Facultad, en los cuales tras administración intravenosa (IV) en la vena braquial del ala, e intramuscular (IM) en el músculo ileotroncal, mediante un diseño cruzado como se observa en el Cuadro 1, de dosis únicas e independientes, de $0,5 \mathrm{mg} / \mathrm{kpv}$, de diazepam y midazolam, se determinó los niveles plasmáticos. La extracción de las muestras se hizo desde la vena yugular derecha. Las especialidades. ensayadas fueron respectivamente diazepam (Valium ${ }^{\circledR}$ ) $10 \mathrm{mg} / 2 \mathrm{~mL}$ sol iny. $\mathrm{y}$ midazolam (Dormicum ${ }^{\circledR}$ ) $15 \mathrm{mg} / 3 \mathrm{~mL}$ sol iny. Se recogieron datos de la respuesta clínica presentada por las aves postadministración ${ }^{4,14}$. Las muestras plasmáticas fueron sometidas a técnica extractiva modificada de Azzam, et al $(1998)^{1,10,11,12}$ y posteriormente analizadas mediante cromatografía líquida de alta resolución (HPLC/UV).

La validación del método se realizó siguiendo las recomendaciones europeas ${ }^{13}$ y de la FDA, analizando los siguientes parámetros: linealidad, rectas de 
calibración, repetitibilidad, reproducibilidad, recuperabilidad y los límites de detección y cuantificación.

Las concentraciones plasmáticas obtenidas fueron sometidas a evaluación farmacocinética con el programa informático PCNONLIN® con el modelo no compartimental. Finalmente los parámetros cinéticos fueron analizados estadísticamente, presentándose los valores con desviaciones estándar (DE) al 95\% de confianza.

\section{RESULTADOS}

Los datos obtenidos se sometieron a un estudio farmacocinético, estadístico y ajustándose a un modelo no compartimental. Los niveles plasmáticos detectados post administración IV de diazepam (Fig. 1), son muy bajos y descienden muy rápidamente no siendo posible procesar los escasos datos de sus metabolitos activos. Los parámetros cinéticos (Cuadro 2), muestran que $t^{1 / 2} \beta$ (semivida de eliminación) es mucho más breve y $\mathrm{Vc}$ (volumen de distribución) y $\mathrm{Cl}$ (depuración) son muy superiores, a los rangos estudiados en perro, conejo $y$ caballo $^{6}$. La absorción de diazepam post administración IM en avestruces es pobre y presenta grandes diferencias individuales, pues sólo obtuvimos una curva de concentraciones en el animal $\mathrm{N}^{\circ} 6$ (AC) (Fig. 2.). Los datos obtenidos de este animal y de los demás, no permitieron su tratamiento cinético. Los valores de $\mathrm{Cl}$ y $t^{1} / 2 \beta$ (Cuadro 3 y 4) de midazolam indican una eliminación más rápida (Fig. 3 y 4) en avestruces que en perros y muy superior a la humana. La biodisponibilidad de midazolam post administración IM es muy alta $(F=99,97 \%)$ similar a la presentada en perros y superior a la biodisponibilidad en humanos, alcanzando rápidamente la concentración máxima (Tmax=11 min). No se detectaron concentraciones plasmáticas del metabolito activo 1 hidroxi-midazolam.

La validación de la técnica analítica empleada para la extracción y determinación de las drogas en plasma nos dieron los resultados que se muestran en el Cuadro 5, según los cuales demostramos que la técnica es confiable para ambas drogas.

\section{DISCUSIÓN}

Los parámetros de validación de la técnica analítica, modificada de Azzam et al. (1998), la hacen confiable y segura. El análisis farmacocinético se hizo con $\mathrm{n}=5$ debido a la elevada desviación de la media que presentó el animal $\mathrm{N}^{\circ} 4$.

Las observaciones clínicas coinciden con los datos de laboratorio. La dosis de $0,5 \mathrm{mg} / \mathrm{kpv}$ (recomendada en la bibliografía) para administración en avestruces domésticos se utilizó como droga única vía IV o IM. La administración de diazepam, por vía IV, a dosis de $0,5 \mathrm{mg} / \mathrm{kpv}$ no se mostró como una opción válida para contención química y sedación de avestruces domésticas juveniles de 6-7 meses de edad. La absorción de diazepam post administración IM en avestruces es errática causando daño tisular en el lugar de administración. Los 
escasos datos obtenidos, tras la administración IM de $0,5 \mathrm{mg} / \mathrm{kpv}$, no permitieron un tratamiento cinético de los niveles plasmáticos detectados.

La administración de midazolam, como agente único a dosis de $0,5 \mathrm{mg} / \mathrm{kpv}$, sí se mostró eficaz pudiendo utilizarse vía IV para obtener inducción anestésica suave, rápida y segura, y por vía $\mathrm{IM}$ para lograr tranquilizaciónb y contención farmacológica eficaz y segura, de los avestruces, durante 10-20 minutos. Las aves no presentaron depresión respiratoria o cardíaca importante que requiriera tratamiento, manteniéndose dentro de los rangos correspondientes al estado de reposo.

Cuadro 1. Diseño Utilizado para la Administración de los Fármacos

\begin{tabular}{|c|c|c|c|c|}
\hline Animal & $\mathbf{1}^{\circ}$ Semana & $\mathbf{2}^{\mathbf{0}}$ Semana & $\mathbf{3}^{\circ}$ Semana & $\mathbf{4}^{\circ}$ Semana \\
\hline NC (1) & DZP IV & MDZ IM & DZP IM & MDZ IV \\
\hline RC (2) & DZP IV & MDZ IM & DZP IM & MDZ IV \\
\hline AC (6) & DZP IV & MDZ IM & DZP IM & MDZ IV \\
\hline AS (3) & MDZ IM & DZP IV & MDZ IV & DZP IM \\
\hline NS (4) & MDZ IM & DZP IV & MDZ IV & DZP IM \\
\hline RS (5) & MDZ IM & DZP IV & MDZ IV & DZP IM \\
\hline
\end{tabular}

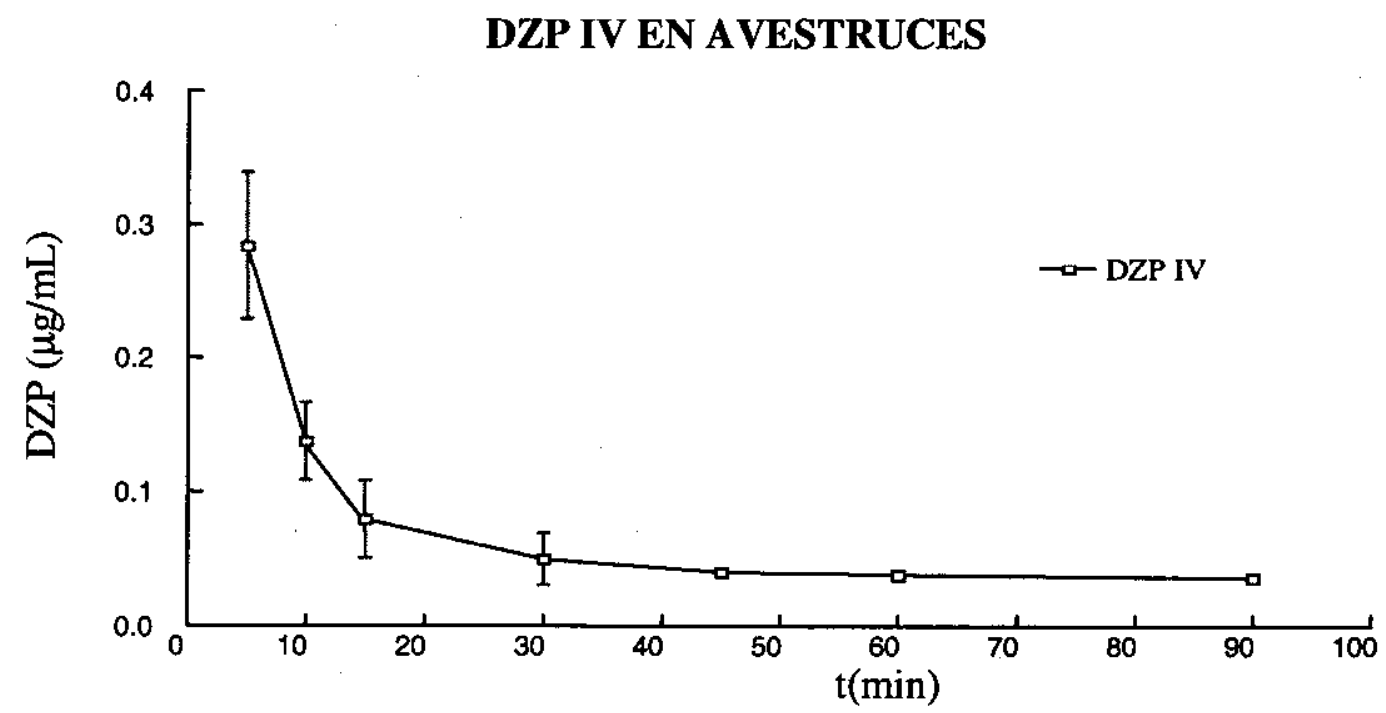

Fig. 1. Gráfica de la evolución de las concentraciones plasmáticas medias $\pm D E$, observadas tras administración IV de diazepam. $(\mathrm{n}=5)$ 
Cuadro 2. Parámetros farmacocinéticos encontrados para diazepam tras administración IV en avestruces

\begin{tabular}{|l|c|c|c|c|c|c|c|}
\hline Parámetro & $1(\mathrm{NC})$ & $2(\mathrm{RC})$ & $3(\mathrm{AS})$ & $\mathbf{4}^{*}(\mathrm{NS})$ & $5(\mathrm{RS})$ & $6(\mathrm{AC})$ & $\begin{array}{c}\text { Media } \pm \mathrm{DE} \\
(\mathrm{n}=5)\end{array}$ \\
\hline $\boldsymbol{\beta}$ & 0,0107 & 0,0525 & 0,0145 & $\mathbf{0 , 0 0 0 8}$ & 0,0111 & 0,0119 & $0,0201 \pm 0,0181$ \\
\hline T 1/2 $\beta$ & 64,6231 & 13,2175 & 47,8285 & $\mathbf{8 8 0 , 8 8 3 7}$ & 62,3767 & 58,4516 & $49,2995 \pm 21,1758$ \\
\hline AUCi & 6,4982 & 2,8612 & 9,1328 & $\mathbf{6 0 , 1 9 5 2}$ & 7,4777 & 8,4629 & $6,8866 \pm 2,4614$ \\
\hline MRTi & 83,5289 & 21,1275 & 67,7176 & $\mathbf{1 2 3 1 , 5 1 6 8}$ & 98,0315 & 85,4373 & $71,1686 \pm 29,9750$ \\
\hline Cli & 76,9 & 17,48 & 54,7 & & 66,9 & 59,1 & $86,5 \pm 50,1$ \\
\hline Vc & 1,4152 & 2,0636 & 1,5533 & & 2,2738 & 1,7768 & $1,8165 \pm 0,3543$ \\
\hline Vssi & 6,4271 & 3,6921 & 3,7074 & & 6,5549 & 5,0449 & $5,0858 \pm 1,3965$ \\
\hline
\end{tabular}

* Animal 4 no incluido para los cálculos por presentar diferencias farmacocinéticas marcadas respecto al grupo.

\section{DIAZEPAM IM EN AVESTRUCES Animal 6(AC)}

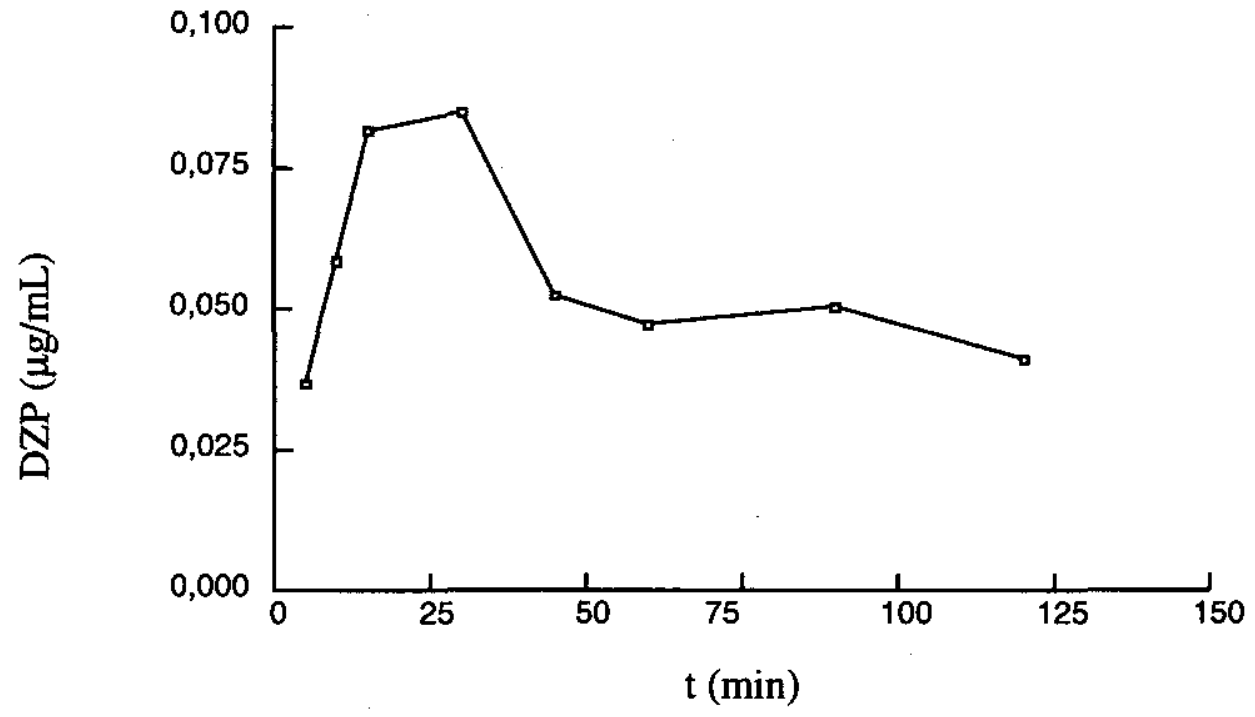

Fig. 2. Gráfica de la evolución de las concentraciones plasmáticas observadas tras administración de diazepam IM. $(n=1)$ 


\section{MIDAZOLAM IV EN AVESTRUCES}

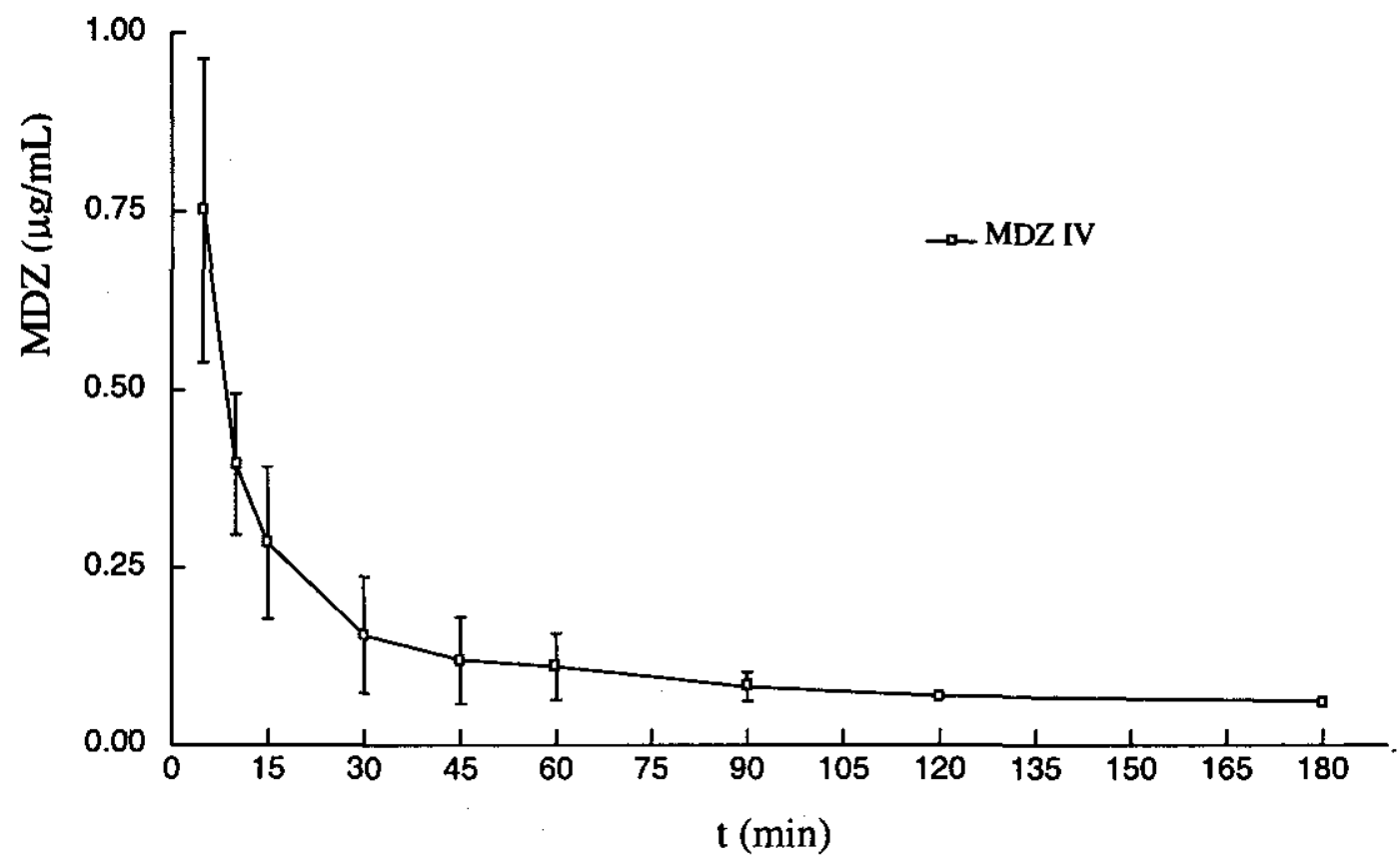

Fig. 3. Gráfica de la evolución de las concentraciones plasmáticas medias $\pm \mathrm{DE}$ observadas tras administración de midazolam IV. $(n=5)$

Cuadro 3. Parámetros farmacocinéticos encontrados para midazolam tras administración IV en avestruces

\begin{tabular}{|l|c|c|c|c|c|c|c|}
\hline Parámetro & $1(\mathrm{NC})$ & $2(\mathrm{RC})$ & $3(\mathrm{AS})$ & $4^{*}(\mathrm{NS})$ & $5(\mathrm{RS})$ & $6(\mathrm{AC})$ & $\begin{array}{c}\text { Media } \pm \mathrm{DE} \\
(\mathrm{n}=5)\end{array}$ \\
\hline$\beta$ & 0,0497 & 0,0614 & 0,0105 & $\mathbf{0 , 0 0 3 2}$ & 0,0124 & 0,0136 & $0,0295 \pm 0,0241$ \\
\hline T $1 / 2 \beta$ & 13,9483 & 11,2917 & 66,2606 & $\mathbf{2 1 4 , 4 5 6 3}$ & 55,8648 & 50,8448 & $39,6420 \pm 25,3038$ \\
\hline AUCi & 6,7244 & 9,4185 & 21,7521 & $\mathbf{4 8 , 3 7 3 3}$ & 28,6184 & 25,9060 & $18,4839 \pm 9,8608$ \\
\hline MRTi & 18,7479 & 20,2312 & 91,1112 & $\mathbf{3 0 6 , 1 6 4 8}$ & 91,1588 & 77,2524 & $59,7003 \pm 37,1459$ \\
\hline Cli & 74,4 & 53,1 & 23,0 & & 17,5 & 19,3 & $37,4 \pm 25,2$ \\
\hline Vc & 0,6571 & 0,7686 & 0,5003 & & 0,5602 & 1,1040 & $0,7180 \pm 0,2385$ \\
\hline Vssi & 1,3940 & 1,0740 & 2,0943 & & 1,5927 & 1,4910 & $1,5292 \pm 0,3709$ \\
\hline
\end{tabular}

* Animal 4 no incluido para los cálculos por presentar diferencias farmacocinéticas marcadas respecto al grupo. 


\section{MIDAZOLAM IM AVESTRUCES}

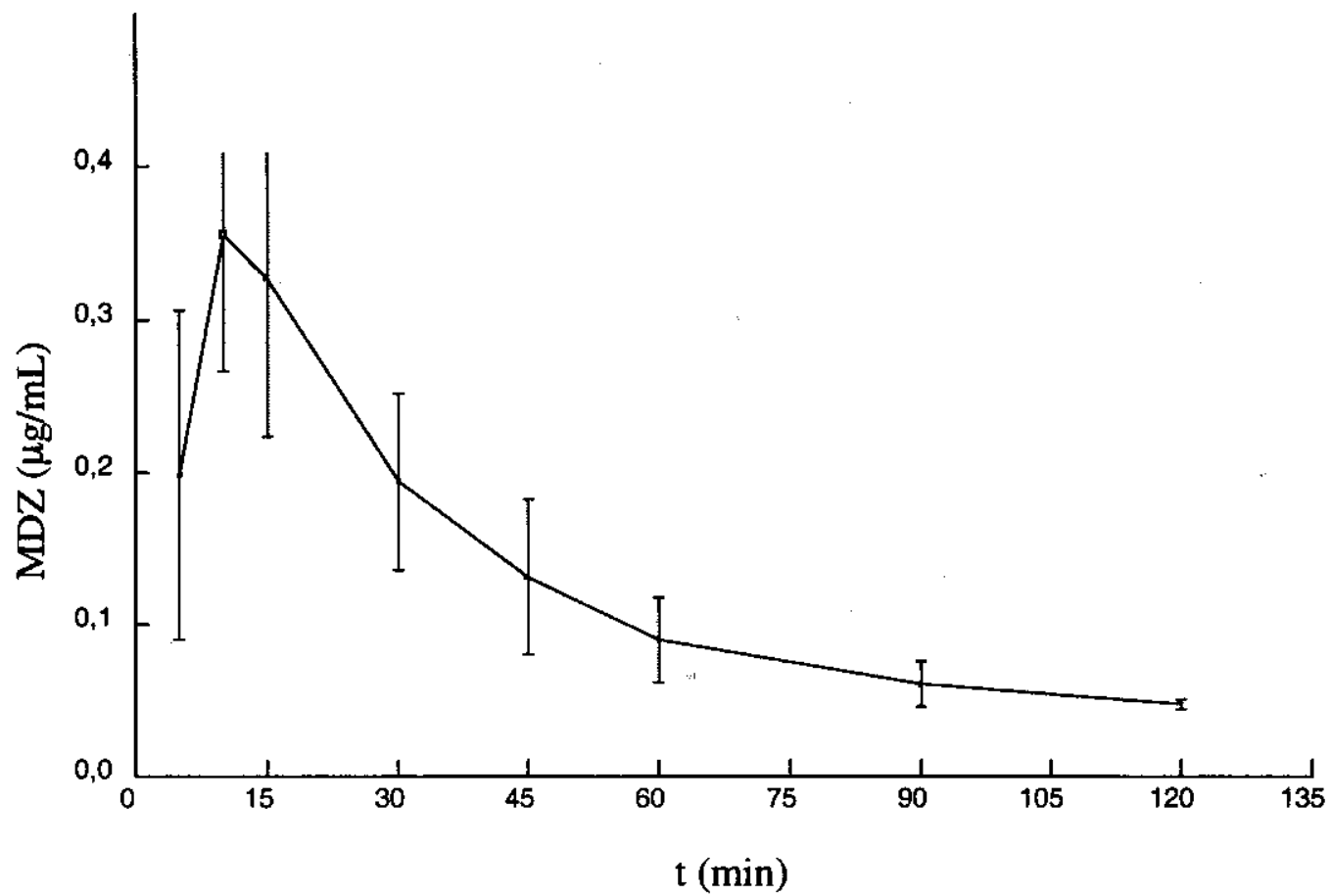

Fig. 4. Gráfica de la evolución de las concentraciones plasmáticas medias $₫ D E$ observadas tras administración de midazolam IM. (n=5)

Cuadro 4. Parámetros farmacocinéticos encontrados para midazolam tras administración $\mathrm{IM}$ en avestruces

\begin{tabular}{|l|c|c|c|c|c|c|c|}
\hline Parámetro & $1(\mathrm{NC})$ & $2(\mathrm{RC})$ & $3(\mathrm{AS})$ & $4^{*}(\mathrm{NS})$ & $5(\mathrm{RS})$ & $6(\mathrm{AC})$ & $\begin{array}{c}\text { Media } \pm \mathrm{DE} \\
(\mathrm{n}=5)\end{array}$ \\
\hline$\beta$ & 0,0235 & 0,0371 & 0,0162 & $\mathbf{0 , 0 0 4 4}$ & 0,0164 & 0,0160 & $0,0218 \pm 0,0091$ \\
\hline t $1 / 2 \beta$ & 29,5196 & 18,7033 & 42,7509 & $\mathbf{1 5 7 , 7 0 3 9}$ & 42,3938 & 43,2272 & $35,3190 \pm 10,9263$ \\
\hline AÚCi & 10,1745 & 9,4946 & 19,7284 & $\mathbf{4 1 , 1 5 6 9}$ & 20,8802 & 21,7874 & $16,4130 \pm 6,0542$ \\
\hline MRTi & 44,6382 & 30,7253 & 61,6069 & $\mathbf{1 7 7 , 0 0 6 6}$ & 59,9108 & 53,5501 & $50,0863 \pm 12,7060$ \\
\hline t máx. & 10 & 10 & 10 & & 15 & 10 & $11 \pm 2,2361$ \\
\hline C máx & 0,2624 & 0,2884 & 0,3597 & & 0,3863 & 0,4895 & $0,3573 \pm 0,0896$ \\
\hline Fi & 151,3072 & 100,8080 & 90,6965 & & 84,1018 & 99,9748 & $99,9748 \pm 30,4282$ \\
\hline
\end{tabular}

* Animal 4 no incluido para los cálculos por presentar diferencias farmacocinéticas marcadas respecto al grupo. 
Cuadro 5. Parámetros de Validación de la técnica analítica para DZP y MDZ.

\begin{tabular}{|l|c|c|}
\hline & DIAZEPAM & MIDAZOLAM \\
\hline $\mathbf{R}^{2}$ en metanol (linealidad) & 0,9925 & 0,9939 \\
\hline $\mathbf{R}^{2}$ en plasma (curva de calibración) & 0,9922 & 0,9906 \\
\hline CV intradía (repetitibilidad) & $7,0 \pm 3,37$ & $5,93 \pm 1,09$ \\
\hline CV interdía (reproducibilidad) & $5,10 \pm 5,47$ & $2,10 \pm 0,26$ \\
\hline Límite de Detección $(\mu \mathrm{g} / \mathbf{m L})$ & 0,0173 & 0,0174 \\
\hline Límite de Cuantificación $(\mu \mathrm{g} / \mathrm{mL})$ & 0,0346 & 0,0347 \\
\hline Porcentaje de Recuperabilidad & $95,29 \pm 5,58$ & $73,07 \pm 6,62$ \\
\hline
\end{tabular}

\section{REFERENCIAS BIBLIOGRÁFICAS}

\section{Azzam RM, Notarianni LJ, Ali HM.} (1998) Rapid and simple chromatographic method for the determination of diazepam and its major metabolites in human plasma and urine. J. Chromatography B. 708 304-309.

2. Blogg SL, Townsend PP, Butler PJ and Taylor EW. (1998) A method of anaesthesia and post operative care for experimental procedures in avian species. An. Technology. 49:2, 101109.

3. Burroughs R. (1996).Capture and Immobilization of Ostriches. International Ratite Symposium. Association of Avian Veterinarians. London.

4. Carbajo E, Castello F, Castello L, Gurri L, Marin M; Mesia J, Sales J, Sarasqueta D. (Nov. 1997) Cría de Avestruces, Emúes y Ñandúes. Real
Escuela de Avicultura. $2^{\mathbf{0}}$ Edición. Barcelona.

5. Cornick JL and Jensen J. (1996) Anesthetic management of Ostriches. Journal of American Veterinary Medical Association 200 (11):16611666.

6. Court MH and Greeblatt DJ. (1992) Pharmacokinetics and preliminary observations of behavioral changes following administration of midazolam to dogs. J. Vet. Pharmacol. Therap. 15 (4), 343-350.

7. De Lucas J.J. (1998) et al. Pharmacokinetics behaviour of ketamine of after intramuscular administration in young ostriches (Struthio camelus). 2nd International Ratite Congress. Oudtshoorn, South Africa . 
8. Huchzermeyer, F. (1995) Patología del Avestruz. Ediciones del Braw Rabós (Girona).

9. Ilkiw JE, Suter CM, Farver TB, Mcneal D \& Steffey EP. (1996) The behaviour of healthy awake cats following intravenous and intramuscular administration of midazolam. Journal of Veterinary Pharmacology and Therapeutics. Vol $19, N^{\circ} 3,217-224$.

10. Jensen J.M. (1994) et al. Metabollic scalling of ketamine in ostriches and emus. Proceedings of the American Association of Zoo Veterinarians. Pittsburg .

11. Mould DR, DeFeo TM, Reele S, Milla G, Limjuco R, Crews T, Choma N, Patel IH. (Jul.1995) .Simultaneous modelling of the pharmacokinetics and pharmacodynamics of midazolam and diazepam. Clin-Pharmacol-Ther.; 58(1): 35-43.

12. Odou P, Robert H, Luycky M, Brunet C, Dine T, Gressier B, Cazin Mand Cazin JC. A Routine HPLC (1997). Method for Monitoring Midazolam in Serum. Biomedical Chromatography vol 11, 19-21.

13. OMCL. (Sept.1999) Validation of Analytical Procedures. PA/PH/OMCL (99) 37, R. Laboratoire National de Santé, Luxembourg.

14. Riviere J.E. (1997) et al. J. Interespecies allometric analysis of the comparative pharmacokinetics of 44 drugs across veterinary and laboratory animal species.

Vet. Pharmacol. Therap. 20: 453 463 